\title{
Poly(vinyl alcohol)/gelatin Hydrogels Cultured with HepG2 Cells as a 3D Model of Hepatocellular Carcinoma: A Morphological Study
}

\author{
Stefania Moscato $^{1, *}$, Francesca Ronca ${ }^{2}$, Daniela Campani ${ }^{2}$ and Serena Danti ${ }^{2,3, *}$ \\ 1 Department of Clinical and Experimental Medicine, University of Pisa, via Savi 10, \\ 56126 Pisa, Italy \\ 2 Department of Surgical, Medical, Molecular Pathology and Emergency Medicine, \\ University of Pisa, via Savi 10, 56126 Pisa, Italy; E-Mails: francesca.ronca@med.unipi.it (F.R.); \\ dcampani@med.unipi.it (D.C.) \\ 3 Laboratory of Creative Engineering \& Design, the Biorobotics Institute, \\ Scuola Superiore Sant'Anna, viale R. Piaggio 34, 56025 Pontedera (PI), Italy \\ * Authors to whom correspondence should be addressed; E-Mails: stefania.moscato@unipi.it (S.M.); \\ s.danti@med.unipi.it (S.D.); Tel.: +39-050-2218617 (S.M.); +39-050997882 (S.D.).
}

Academic Editor: Sue Anne Chew

Received: 17 November 2014 / Accepted: 5 January 2015 / Published: 13 January 2015

\begin{abstract}
It has been demonstrated that three-dimensional (3D) cell culture models represent fundamental tools for the comprehension of cellular phenomena both for normal and cancerous tissues. Indeed, the microenvironment affects the cellular behavior as well as the response to drugs. In this study, we performed a morphological analysis on a hepatocarcinoma cell line, HepG2, grown for 24 days inside a bioartificial hydrogel composed of poly(vinyl alcohol) (PVA) and gelatin (G) to model a hepatocellular carcinoma (HCC) in 3D. Morphological features of PVA/G hydrogels were investigated, resulting to mimic the trabecular structure of liver parenchyma. A histologic analysis comparing the 3D models with HepG2 cell monolayers and tumor specimens was performed. In the 3D setting, HepG2 cells were viable and formed large cellular aggregates showing different morphotypes with zonal distribution. Furthermore, $\beta$-actin and $\alpha 5 \beta 1$ integrin revealed a morphotype-related expression; in particular, the frontline cells were characterized by a strong immunopositivity on a side border of their membrane, thus suggesting the formation of lamellipodia-like
\end{abstract}


structures apt for migration. Based on these results, we propose PVA/G hydrogels as valuable substrates to develop a long term 3D HCC model that can be used to investigate important aspects of tumor biology related to migration phenomena.

Keywords: three-dimensional (3D); cancer model; poly(vinyl alcohol) (PVA); gelatin; HepG2; hepatocellular carcinoma (HCC); histology; cell morphology

\section{Introduction}

Hepatocellular carcinoma (HCC) accounts for $70 \%-80 \%$ of the total liver cancers and many efforts have been made in the latest decades to find new and more efficient therapies to prolong the overall survival of patients [1,2]. Systemic chemotherapy after surgery, adjuvant therapies and new molecular targeted drugs have guaranteed only a partial success due to their limited case application. Moreover, as in other cancer treatments, also in HCC drug resistance represents a major challenge [3,4]. Liver transplantation is considered the last therapeutic option owing to the low number of donors, the use of immunosuppressive drugs, which can cause severe collateral effects, and the high frequency of both viral and bacterial infections $[3,4]$. It appears thus evident that innovative therapies, more effective, selective and possibly patient-specific, are greatly expected.

In vitro models have been developed to allow the study of tumor cell behavior in response to therapies [5]. The bidimensional (2D) cultures have made it possible to investigate different cellular phenomena such as proliferation, differentiation or function. Anyway, in tissues or organs, cells are naturally organized in three-dimensional (3D) structures interacting with both other cell types and the surrounding extracellular matrix (ECM). As a consequence, inherent limits of 2D models, such as cell phenotype and ECM dissimilarities with respect to those of the tumor and insufficient microenvironment complexity, have revealed to affect the reliability of these models [6,7]. To overcome such difficulties, new 3D in vitro models have been recently proposed [8-10]. Despite that, so far the pharmacological studies to evaluate the cytotoxicity or the pharmacokinetic of new drug compounds have been mainly performed on 2D models.

In normal (i.e., non-cancerous) cell cultures, phenotype and genotype differences have been documented between $2 \mathrm{D}$ and $3 \mathrm{D}$ in vitro models, so as many other biological phenomena including cell proliferation, differentiation or function [11]. Some authors have highlighted that these differences are even more profound when cancer cells are cultured in a $3 \mathrm{D}$ structure compared to a monolayer $[12,13]$. These findings rendered it appealing the consolidation of the tissue engineering approaches to understand tumor cell dynamics involved in proliferation, metastasis and pharmacological response [7,10,14].

Indeed, in cancer applications, tissue engineering offers innovative options focusing on the study of new biomimetic and biocompatible materials that can support the proliferation and the viability of cells to create not only normal, but also pathological 3D tissue models $[15,16]$. The development of advanced $3 \mathrm{D}$ in vitro models can disclose the comprehension of tumor progression and behavior in different experimental conditions. One of the key factors that influence successful outcomes in the field of tissue engineering is the identification of an appropriate scaffold that resembles the selected in vivo environment. 
Some tissue engineering approaches have been used by researchers to create bio-hybrid constructs as a 3D model of HCC. Scaffolds both of synthetic and biologic origins have been investigated for the growth and function of HepG2 cells, the latter considered as a consolidated cellular model of HCC. The synthetic matrices tested for 3D culture of HepG2 included beads of apatite or silica, porous polymeric scaffolds based on poly(vinyl alcohol) (PVA), tetraethoxysilane/polydimethylsiloxane, polyurethane, and polystyrene [13,17]. In these scaffolds, the HepG2 cells generally revealed an enhanced metabolic activity compared to the $2 \mathrm{D}$ conditions. Moreover, these findings suggested that the 3D environment strongly influenced drug efficacy, thus resembling more closely the tumor phenomena occurring in vivo [13]. Among the biological materials, proteins and polysaccharides have been used to prepare scaffolds. Matrigel ${ }^{\circledR}$, collagen and self-assembled peptide nanofibers have been reported in some studies, showing both enhanced hepatic function and promotion of malignant phenotype in HepG2 cells, with respect to 2D cultures [18,19]. Finally, 3D HCC models have been fabricated via HepG2 encapsulation inside alginate hydrogels [20]. In these culture conditions, the cells were not able to proliferate, even if they were found to be viable and functionally active. This phenomenon was supposed to be a consequence of ineffective cell adhesion to the biomaterial matrix.

Hydrogels represent an intriguing class of biomaterials for scaffold fabrication, owing to the high water content that they can retain, the similarity with the ECM and their moldability into plenty of different shapes [21-23]. In particular, PVA is a synthetic polymer that can form hydrogels under a physical cross-linking, obtained by repeated freeze-thawing cycles of aqueous polymeric solutions [24]. Bioartificial hydrogels, combining PVA with different biologic molecules, such as dextran, hyaluronic acid and gelatin (G), have been proposed for tissue engineering applications [25]. Such hydrogels for certain weight composition ratios between the biological and synthetic components displayed anisotropic lamellar structures and small size macropores that could represent interesting features to mimic the liver structures.

This study was aimed at developing a novel 3D HCC model to enable the comprehension of biological processes involved in tumor cell migration, focusing on the morphological aspects of cancer cells. We prepared PVA/G hydrogels with weight composition 80/20 under sterile conditions. The morphology of PVA/G scaffolds was analyzed via scanning electron microscopy (SEM) and mercury intrusion porosimetry. These hydrogels were seeded with HepG2 cells and cultured in vitro up to 24 days. Viability of cell/scaffold constructs was monitored along the culture time using a metabolic activity assay, and double-checked at the endpoint through the expression of hypoxanthine-guanine phosphoribosyltransferase (HPRT), as a fundamental metabolic enzyme, via western blotting. The morphology of cell/scaffold constructs was analyzed via histology and compared to those of HepG2 cells in 2D cultures and HCC specimens. Finally, the expression of $\beta$-actin, investigated via immunohistochemistry and western blotting, and the immunolocalization of $\alpha 5 \beta 1$ integrin were evaluated and put in relation to HepG2 cell morphology.

The establishment of an HCC model mimicking cancer cell migration and invasion is expected to give new insight on the tumor biology, thus paving the way to the research and development of more effective therapies. 


\section{Results}

\subsection{Morphological Characterization of PVA/G Hydrogels}

Macroporous hydrogels were prepared under sterile conditions blending water solutions of PVA and $\mathrm{G}$ with culture medium (CM) and using freeze-thawing cycles to obtain the physical crosslinking of PVA. The hydrogels assayed in this study had a final PVA/G ratio of $80 / 20(\mathrm{w} / \mathrm{w})$, a $93.5 \%$ weight water content and a $425 \%$ volume increase. SEM analysis of PVA/G hydrogels showed a porous 3D structure arranged as thin directional lamellar structures (Figure 1a). Size and distribution of macropores were investigated via mercury intrusion porosimetry. Data were reported as the percentage of volume occupied by pores whose diameter ranged in selected classes (Figure 1b). The pores with diameters ranging in 10-20 $\mu \mathrm{m}$ seized the biggest volume fraction $(36.82 \% \pm 3.12 \%)$. On the whole, $71.59 \% \pm 5.03 \%$ of the measured volume was due to pores with diameters lower than $30 \mu \mathrm{m}$ (namely, ranging in $0.007-30 \mu \mathrm{m}$ ), while only $28.41 \% \pm 4.57 \%$ of the measured volume derived from pores with larger diameters (namely, ranging in 30-150 $\mu \mathrm{m}$ ).

(a)

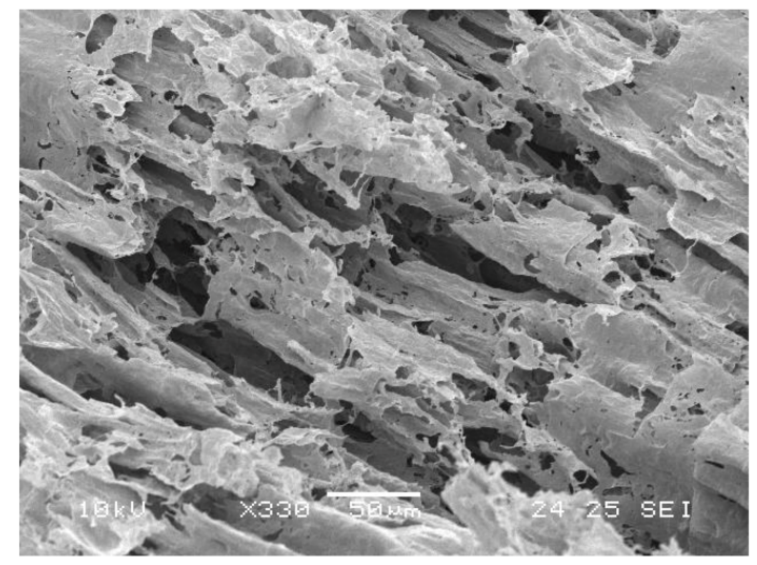

(b)

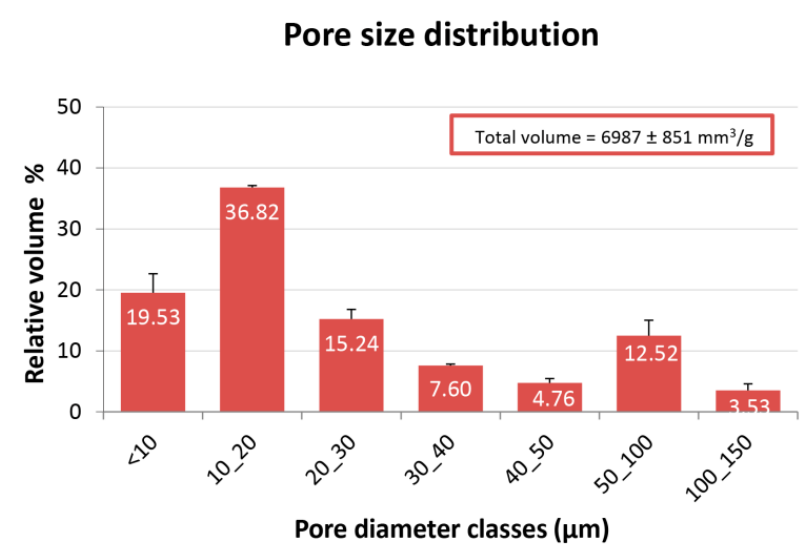

Figure 1. Morphological analysis of PVA/G scaffolds: (a) SEM micrograph showing the inner structure of the scaffold; (b) bar graph showing the porosimetric analysis of the scaffold, expressed as relative volume percent for selected poral classes. Data are reported as mean $\pm \mathrm{SD}$.

\subsection{Viability of Cell/Scaffold Constructs}

The alamarBlue ${ }^{\circledR}$ assay was used to monitor the metabolic activity of HepG2 cells along the culture time as a proof of their viability within the scaffolds (Figure 2). The bioassay revealed that the cells were viable up to 24 days in culture inside PVA/G hydrogels.

The metabolic activity, expressed as dye reduction percent $\left(\% \mathrm{AB}_{\mathrm{red}}\right)$, measured in cell/scaffold constructs on day $3,10,17$ and 24 after seeding, was $24.35 \% \pm 4.54 \%, 22.99 \% \pm 3.82 \%, 29.48 \% \pm 6.58 \%$ and $42.12 \% \pm 7.53 \%$, respectively. At the endpoint, the metabolic activity of the constructs resulted significantly higher than that detected at all the previous time-points, showing $p$ values equal to 0.0019 , 0.0005 and 0.0013 in comparison against day 17, 10 and 3, respectively. No statistically significant differences were revealed when the day 3, 10, 17 time-points were compared among each other. 


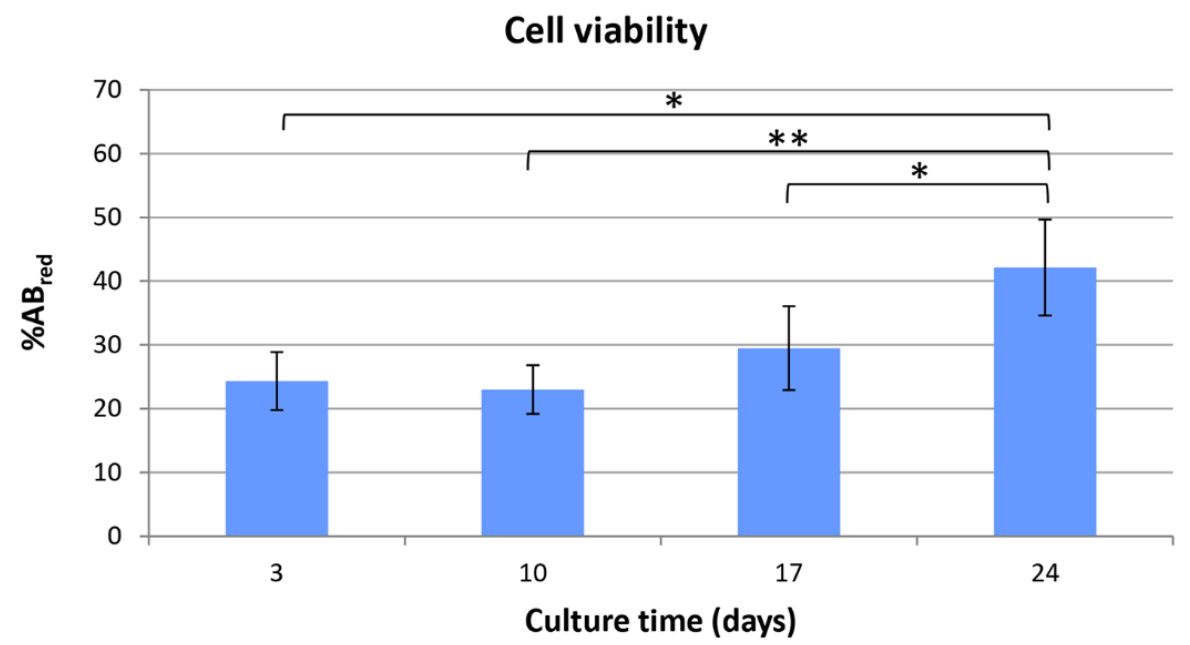

Figure 2. Bar graph reporting the results of alamarBlue ${ }^{\circledR}$ assay at different time-points. Data are reported as mean $\pm \mathrm{SD}$; asterisks indicate the following magnitude orders of $p$ values: $* 10^{-3}$ and $* * 10^{-4}$.

\subsection{Histologic Analysis}

Morphological analysis of cellular samples were performed using hematoxylin and eosin (H\&E) staining (Figure 3a,c,e), while Periodic Acid Shiff (PAS) reaction was used to highlight the presence of glycoproteins and glycogen, as a product of metabolically active hepatic cells (Figure 3b,d,f). Glycoproteins are a class of adhesive proteins that own binding sites enabling cell attachment to the ECM. Specifically, histologic analyses were performed on HepG2 cells, samples of HCC and HepG2 cells cultured inside PVA/G hydrogels. In 2D cultures, HepG2 cells showed a typical stellate shape when distributed at a low density, while a round shape when assembled in cluster-like formations (Figure 3a). In both morphotypes, PAS reaction revealed a weak positivity as only a few cytoplasmatic granules were stained (Figure 3b). The PAS positivity was also observed on tumor sections, in which most cells displayed diffuse staining, while only a reduced number of cells were strongly positive (Figure 3d).

Both PAS reaction and H\&E staining showed that hepatocytes reorganized into duct-like formations. Such a differentiative parameter is a hallmark of the HCC tumor (Figure 3c,d). In cell/scaffold constructs, H\&E highlighted that HepG2 sprout out inside the hydrogels forming large cellular aggregates within the polymeric matrix. These cellular structures were constituted by different morphotypes according to their localization and three main areas could be recognized (Figure 3e,f). In the central area, indicated as S1, small cells with a big nucleus and an eosinophil cytoplasm were present and arranged to form trabecular-like structures. The peripheral area, indicated as S3, was populated mainly by large cells, strongly basophilic with a flat shape, suggesting a stellate-like morphology.

Furthermore, in this area, PAS reaction was strongly positive both at intra- and extra-cellular level, thus denoting an active secretion of glycoproteins. Between S1 and S3, an intermediate area could be observed, indicated as S2, in which cells displayed pyknotic nuclei and damaged membranes, as a probable result of cellular stress. 

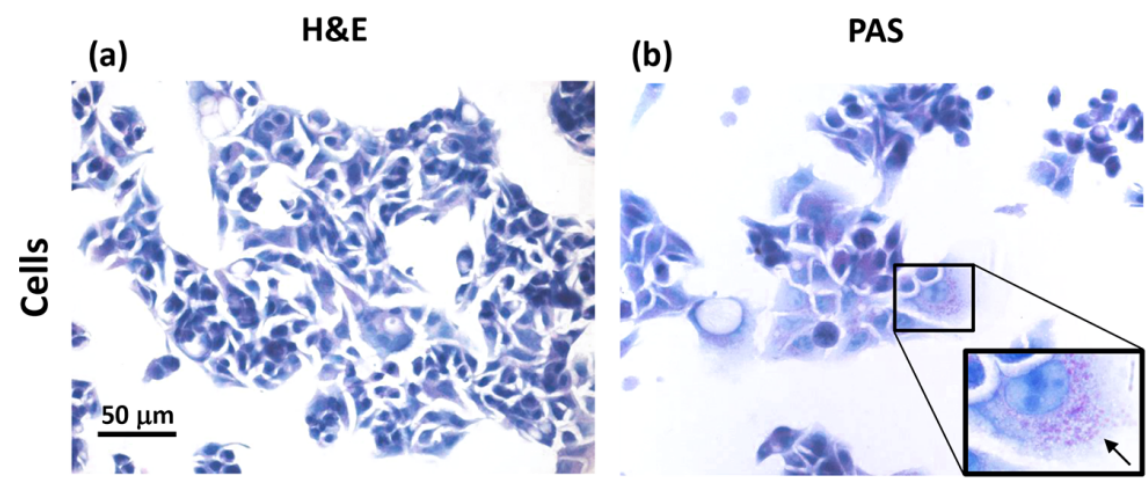

(c)

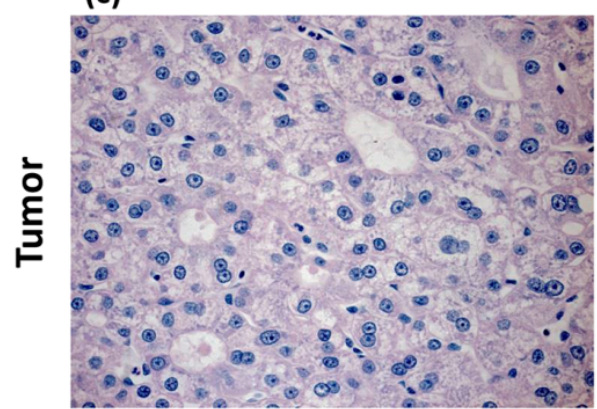

(d)

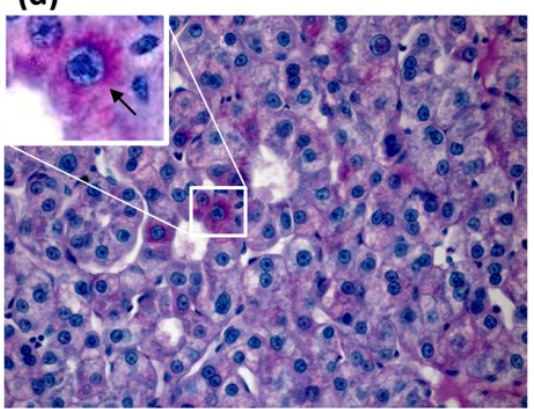

(e)

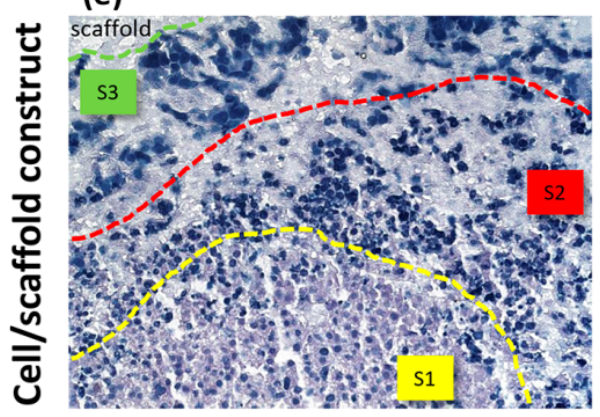

(f)

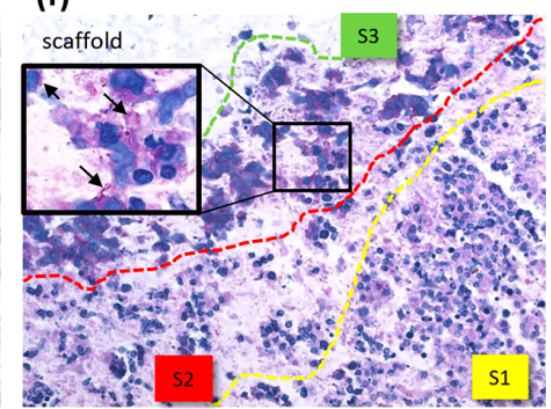

Figure 3. Histochemical analysis of HepG2 cells cultured in monolayers (a,b); samples of HCC (c,d) and HepG2 cells cultured inside PVA/G hydrogels (e,f). For each sample type, $\mathrm{H} \& \mathrm{E}(\mathrm{a}, \mathrm{c}, \mathrm{e})$ and PAS reaction $(\mathrm{b}, \mathrm{d}, \mathrm{f})$ are shown. Arrows in $(\mathrm{b}, \mathrm{d}, \mathrm{f})$ indicate evidence of PAS positivity. S1, S2 and S3 in (e,f) define the areas of different morphotype localization in cell/scaffold constructs.

In this study, two fundamental proteins related to cell migration were evaluated, namely $\beta$-actin and $\alpha 5 \beta 1$ integrin. $\beta$-actin, a protein involved in cytoskeleton arrangement and remodeling, was investigated via immunohistochemistry and compared to negative controls (CTRL) performed omitting the primary antibody (Figure 4).

HepG2 cells cultured in monolayers showed a high immunopositivity for $\beta$-actin with a filamentous pattern distribution (Figure 4b). Differently, in tumor sections, $\beta$-actin was preferentially localized under the plasma membrane and in the correspondence of duct lumen, in which cell microvilli protruded (Figure 4d). This immunohistochemical observation was corroborated by the presence of zonal cell distribution in the cell/scaffold constructs, highlighting the presence of different cellular morphotypes identifiable via histochemical staining (Figure 4e,f). The small cells in the central area (S1) were more immunopositive to $\beta$-actin than the large cells located in the peripheral area. However, the stellate cells 
showed a strong and specific immunoreaction localized on a side edge of their membrane (insert in Figure 4f).

(a)

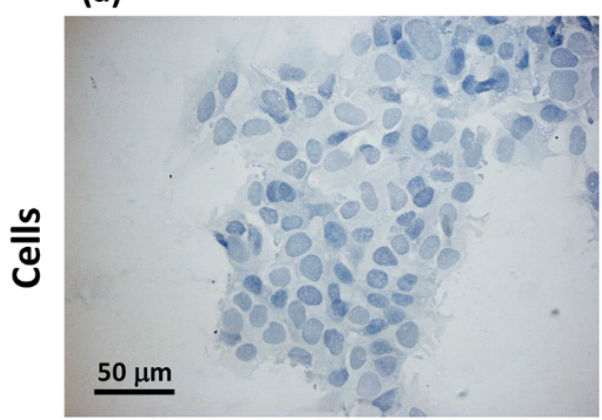

(c)

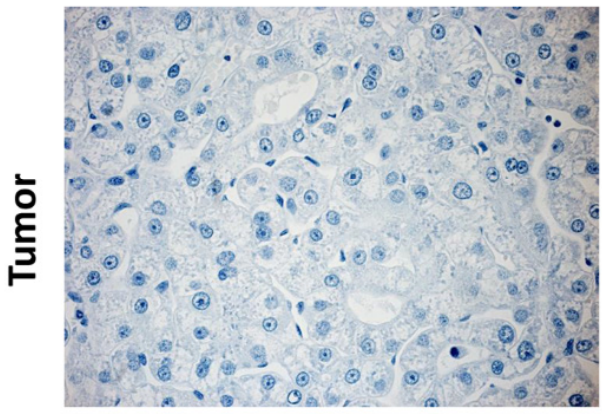

(e)

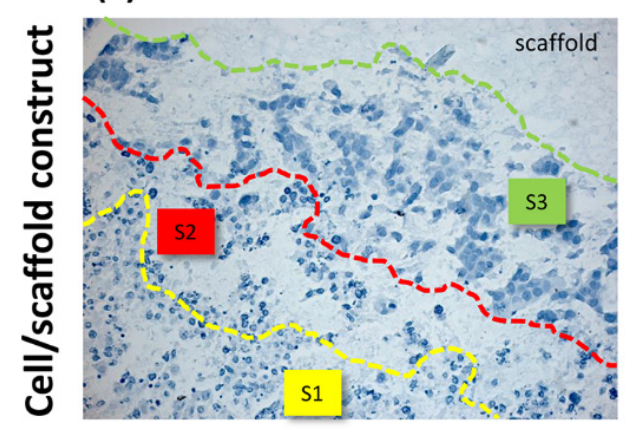

(b)

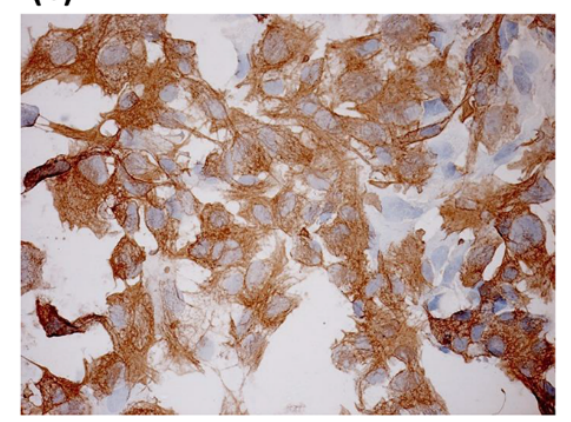

(d)

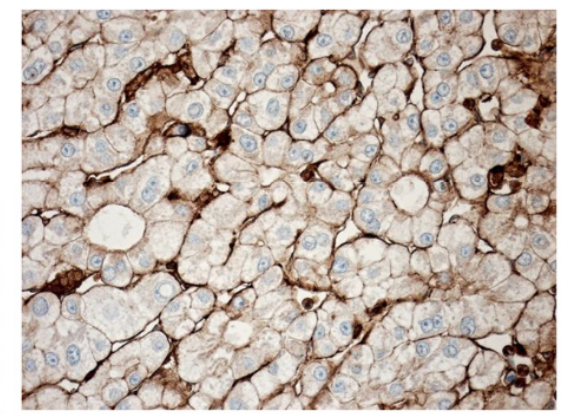

(f)

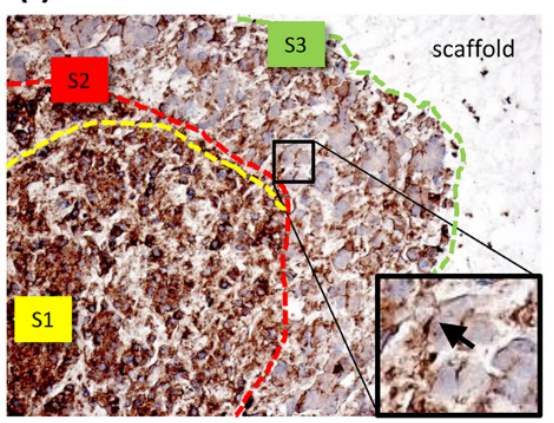

Figure 4. Immunohistochemical analysis of HepG2 cells cultured in monolayers (a,b); samples of HCC (c,d) and HepG2 cells cultured inside PVA/G hydrogels (e,f). For each sample type, negative controls (a,c,e) and $\beta$-actin expression $(b, d, f)$ are shown. S1, S2 and $\mathrm{S} 3$ in $(\mathrm{e}, \mathrm{f})$ define the areas of different morphotype localization within the cell/scaffold constructs. The insert in (f) shows a few cells with a lamellipodial-like expression of $\beta$-actin, indicated with an arrow.

Immunohistochemistry revealed a similar expression pattern for $\alpha 5 \beta 1$ integrin, a cell-matrix transmembrane receptor binding ECM and proteinases expressed by HepG2 cells. The results, showing specific immunopositivity of $\alpha 5 \beta 1$ integrin on the side border of cells lying in the S3 zone, are displayed in Figure 5. The anti-integrin $\alpha 5$ antibody here used recognized the intracellular (i.e., cytoplasmatic) domain of the protein, thus highlighting morphological features of the cells. 
(a)

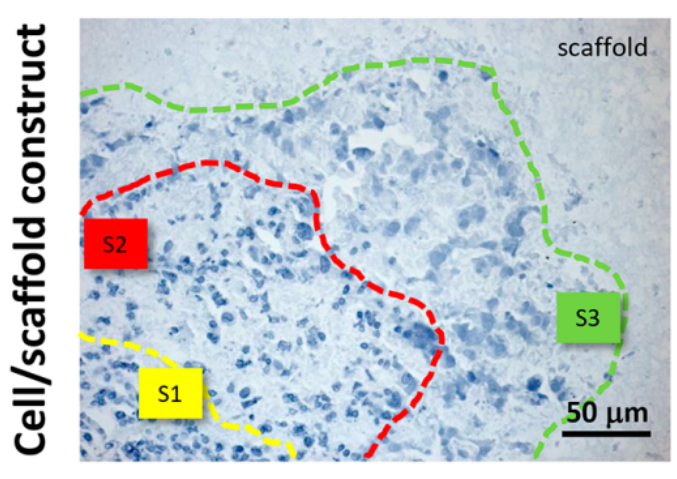

CTRL

(b)

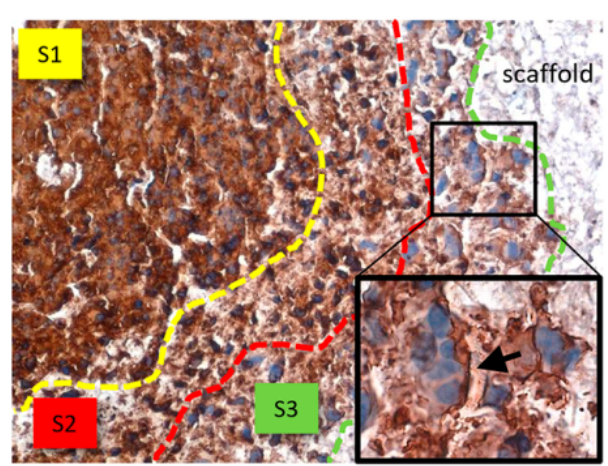

Figure 5. Immunohistochemical analysis of HepG2 cells cultured inside PVA/G hydrogels, showing negative control and $\alpha 5 \beta 1$ integrin expression $(\mathbf{a}, \mathbf{b}) . \mathrm{S} 1, \mathrm{~S} 2$ and S3 in $(\mathrm{a}, \mathrm{b})$ define the areas of different morphotype localization within the cell/scaffold constructs. The insert in (b) shows a few cells with a side border expression of $\alpha 5 \beta 1$, indicated with an arrow.

\subsection{Western Blotting}

Immunoblot assay was performed on HepG2 cells cultured both in monolayers (2D) and inside PVA/G hydrogels (Figure 6). Results showed a high expression of $\beta$-actin in cell/scaffold constructs, thus confirming the previous immunohistochemical data. Moreover, the volume intensity evaluation of the bands revealed that in the cell/scaffold constructs $\beta$-actin was expressed at a higher concentration than in HepG2 monolayer cultures, taking in account that the same total protein amount was loaded and separated by the SDS-PAGE gels. Its calculated values for band volume intensity of cell/scaffold constructs and monolayer cells were $41.10 \times 10^{5}$ and $32.06 \times 10^{5}$, respectively (Figure 6b).

(a)

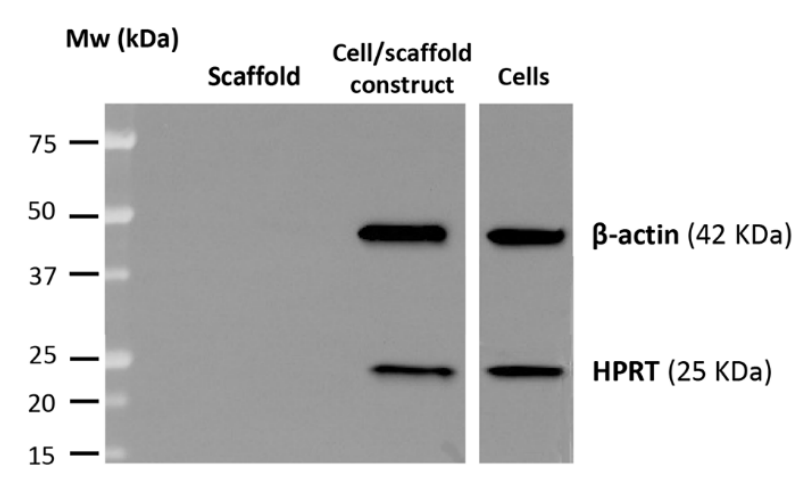

(b)

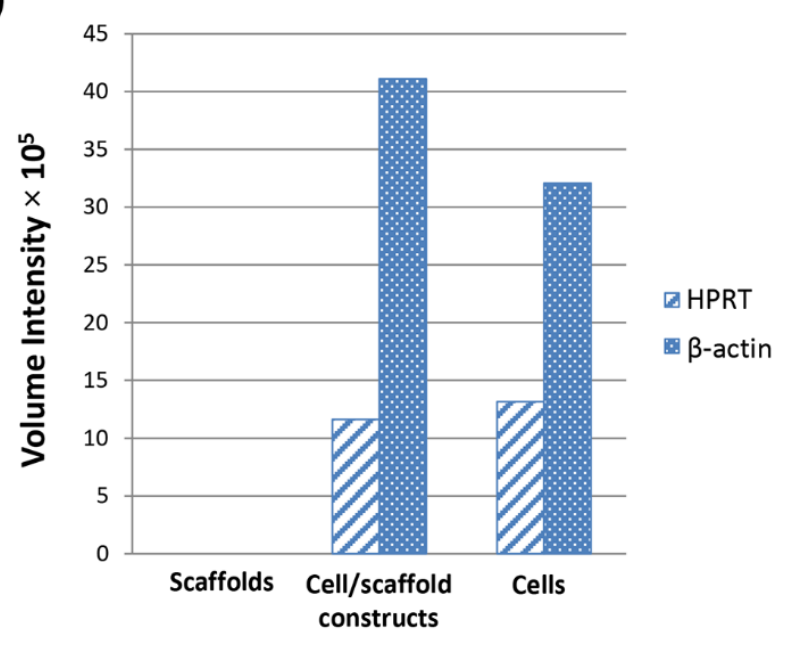

Figure 6. Western blot results of $\beta$-actin and HPRT expression in HepG2 cells cultured in monolayers for 4 days ( $80 \%$ confluence) and inside PVA/G hydrogels for 24 days. Hydrogels without cells were tested as negative controls. The immunoblot reaction is shown in (a) and the band analysis in (b). 
HPRT was evaluated via immunoblotting as a marker of metabolic activity (Figure 6). The band volume intensities showed a reading of $11.61 \times 10^{5}$ and $13.16 \times 10^{5}$, for cell/scaffold constructs cultured for 24 days and monolayer cells cultured for 4 days (up to $80 \%$ confluence), respectively (Figure 6b). No appreciable difference in the HPRT expression was revealed between the two culture conditions.

\section{Discussion}

In this report, we propose a new 3D in vitro model of $\mathrm{HCC}$ based on HepG2 liver cells seeded inside PVA/G hydrogels and cultured for long term (namely, 24 days) to provide a platform that will potentially enable the study of biological processes driven by the migratory capability of cancer cells. We performed a morphological investigation as a preliminary study to reveal migration-related changes of cell morphology inside a 3D environment. Our results demonstrated that HepG2 cells modified their morphological features and cytoskeletal organization according to their zonal localization within the cellular aggregates formed inside the scaffolds. These findings highlighted the importance of the 3D microenvironment for the comprehension of the mechanisms occurring in tumor progression.

Some authors have reported difficulties related to the use of hydrogels as scaffolds, owing to some practical issues, such as gel preparation, storage and variability [13]. Differently, PVA/G hydrogels are easy to prepare [24,25]. Additionally, they can be produced as sterile substrates, since the polymer is dissolved by autoclaving and the gelatin aqueous solution at the used concentration can be sterile-filtered. Moreover, the freeze-thawing procedure can be performed using sealed plates in order to preserve the material sterility. These bioartificial hydrogels combined the structural properties of the synthetic polymer (PVA) with the high cell affinity of the protein (G), thus enhancing the scaffold cytocompatibility [25]. Moreover, it has been documented that blending PVA with G up to $20 \%$ (w) to form hydrogels gave rise to directional lamellar structures [25]. To dilute the initial PVA/G mixture up to the desired final PVA concentration, complete CM was added during preparation. In this way, the hydrogels obtained were ready to use as scaffolds, since they already incorporated cell culture supplements and provided cell suitable osmolarity in the liquid phase. The proposed method for hydrogel preparation can be easily afforded by biological laboratories that are not equipped with scaffold fabrication facilities, thus representing a user-friendly procedure to prepare substrates for 3D cancer models.

From a morphological point of view, PVA/G scaffolds appeared to be as macroporous substrates with anisotropic lamellar structures outlined by thin mesoporous polymeric surfaces. Such a scaffold morphology reminded the trabecular structure of liver parenchyma and appeared suitable for liver cell disposal. In the dried hydrogels, about $3 / 4$ of the empty volume derived from pores with diameters lower than $30 \mu \mathrm{m}$, while greater pore diameter classes were present at a lower extent. These results were in line with previous observations [25]. It has been documented that the poral features of the scaffolds play a key role in cell colonization, morphology and function [26]. Moreover, the scaffold inner architecture, as a result of spongy or nanofiber-interspace pores, can be a discriminant in cancer modeling [17,27]. Plain PVA porous scaffolds, as substrates for HepG2 cells, have been firstly proposed by Kataoka et al. [17]. In this study, commercial PVA scaffolds with $130 \mu \mathrm{m}$ and $200 \mu \mathrm{m}$ were investigated and compared with other polymeric scaffolds with similar porosity. The authors reported a reduced cell proliferation and colonization that were attributed to the surface properties of PVA, supposed to impair an efficient cell clustering. The presence of biological macromolecules in PVA hydrogels has been reported to modify 
their internal architecture and possibly their surface properties [25]. As an example, PVA/G in the form of sponges, has been documented to act as a valuable support for fibroblast growth and ECM synthesis [28]. In our experimental conditions, the use of bioartificial PVA hydrogels with pore size lower than that described by Kataoka et al. resulted well suitable for HepG2 colonization. However, it has to be outlined that pore size distribution, as conventionally measured on dried samples, can be only representative of materials, such as hydrogels, that meet the target of swelling. After preparation, PVA/G hydrogels appeared as chewy gels with a strong capacity of liquid retention as demonstrated by a $93.5 \%(\mathrm{w})$ weight water content and a $425 \%$ volume increase. In the first instance, this aspect challenged the cell seeding events. However, Hamilton syringes made it possible to perform cell seeding via inoculation with an acceptably low variability, which was confirmed by the outcomes of alamarBlue ${ }^{\circledR}$ assay. Indeed, the HepG2 cells seeded inside PVA/G hydrogels were able to maintain their metabolic activity for more than 2 weeks and showed a statistically significant increase at the endpoint. At that time, the occurrence of neither apoptotic nor highly relevant necrotic phenomena could be documented (data not shown). Therefore, it cannot be excluded that the cells underwent a lag stage followed by an intense proliferation phase. The metabolic activity outcome was corroborated by the expression of HPRT, revealed via western blotting. HPRT is a transferase that catalyzes the conversion of hypoxanthine to inosine monophosphate, and guanine to guanosine monophosphate. This enzyme plays an important role in the generation of purine nucleotides throughout the purine salvage pathway. The presence of HPRT in the constructs confirmed active cell metabolism after 24 days in culture.

Other studies have evaluated several devices, fabricated using synthetic or biological materials for HepG2 3D culture, also in dynamic conditions [13,17-20]. However, these investigations were performed at earlier culture times, i.e., up to 14 days. Moreover, an extensive morphological analysis of cell/scaffold constructs has not been reported so far. Indeed, Bokhari et al. have shown morphological features of HepG2 cells in polystyrene scaffolds via electron microscopy [13]. Similarly, Kataoka et al. have reported cell presence in the constructs via SEM and basic histologic analysis (H\&E) [17]. In our opinion, histology represents a valuable tool to highlight important phenomena, such as tumor cell migration, that can be more efficiently observed in 3D models at later culture times.

Many authors have referred to spheroids as 3D cancer models [29]. However, these structures have been documented to mimic only some aspects of the tumor microenvironment [7,30]. Tissue-engineered constructs have been recently proposed as more reliable cancer models than spheroids $[7,10,16,30]$. Indeed, if cultured inside biomaterial scaffolds, tumor cells are more likely to reproduce the in vivo tissue architecture and be viable for extended periods [13,27]. In our investigation, we performed a histologic analysis to detect cell morphology and the expression of biosynthesis, structural and interaction molecules, such as glycoproteins, $\beta$-actin and $\alpha 5 \beta 1$ integrin. In the cell/scaffold constructs, cells were found to be arranged in large aggregates, either located at the inoculation spots or in separate sites of the hydrogel, that were all characterized by three different areas, in which diverse HepG2 morphotypes were present. In the central area, small cells were arranged to form trabecular-like structures, similar to the liver parenchyma, while the peripheral area was populated by large stellate cells with an active secretion of glycoproteins. In between these two areas, stressed cells and a few necrotic phenomena were observed. The presence of a necrotic zone has been detected also in tumor spheroids, being preferentially localized in the core as a critical area of metabolite distribution [31]. Differently, our constructs showed distinct cellular agglomerates, each one characterized by some evidence of necrotic cells only in an intermediate 
layer between two different cell morphotype areas. This phenomenon thus seemed not to be a consequence of metabolite gradients.

Unlike the cells localized in the most internal layers of the cellular aggregates, which showed the highest and uniformly distributed $\beta$-actin and $\alpha 5 \beta 1$ integrin expression, the cells in the outer layer displayed a specific $\beta$-actin and $\alpha 5 \beta 1$ integrin immunopositivity on a side border of their membrane, thus suggesting the formation of lamellipodia-like structures. The regulation and reorganization of cytoskeleton proteins, such as $\beta$-actin, has been invoked as a key factor in cancer cell migration, through the formation of lamellipodia and invadopodia/podosomes [32]. Lamellipodia are the main cellular structures devoted to the cell-substrate interactions, enabling the driving force for cell migration induced by chemoattractant gradients [32]. This fact is corroborated by a similar localization of $\alpha 5 \beta 1$ integrin in our samples. On the basis of these data, our findings suggested that the stellate cells on the frontline layers of cellular aggregates were interacting with the PVA/G hydrogel surfaces modifying their cytoskeletal filaments and triggering their connections with the outer ECM molecules in order to migrate across the scaffold pores.

Comparing to HepG2 monolayers, cell/scaffold constructs represented an advanced model of HCC. Indeed, in 2D cultures, the territorial difference between the two morphotypes was not highly marked and no cytoskeletal reorganization was observed. The comparison of the 3D model with HCC specimens appeared more difficult, since the tumor tissues analyzed in this study showed well defined and mature characteristics typical of a late differentiation stage. In these tumor sections it was not possible to observe dynamic phenomena, such as cell migration.

All in all, PVA/G hydrogels appeared to promote the long-term growth and the differentiation of the hepatocarcinoma cell line HepG2, allowing the formation of more complex 3D structures that can be useful to study the biology of HCC tumors. In particular, these 3D constructs could be tested for drug susceptibility and possibly give new insight to target, develop or refine cancer therapies. These bioartificial scaffolds are thus proposed as a valuable tool to develop a long term 3D model of HCC.

\section{Experimental Section}

\subsection{Preparation of PVA/G Hydrogels}

PVA/G hydrogels were prepared modifying a procedure described in a previous study [25] in order to obtain sterile scaffolds ready to use. Briefly, a $10 \%(\mathrm{w} / \mathrm{v})$ solution of PVA $(99 \%$ hydrolyzed, $M_{\mathrm{w}}$ 85,000-146,000; Sigma-Aldrich, Milan, Italy) in distilled water was prepared using an autoclave cycle, set at $120{ }^{\circ} \mathrm{C}$ and $20 \mathrm{~min}$. The PVA solution was cooled down to $60{ }^{\circ} \mathrm{C}$ under magnetic stirring, opened inside a cell culture flow cabinet and blended with a $3 \%(\mathrm{w} / \mathrm{v})$ sterile filtered solution of gelatin (G; from skin bovine type B, 75 Bloom; Sigma-Aldrich) in distilled water to obtain a bioartificial mixture with a PVA/G ratio of $80 / 20(\mathrm{w} / \mathrm{w})$. This mixture was complemented with complete $\mathrm{CM}$, consisting of red phenol-free Dulbecco's modified Eagle Medium (D-MEM) supplemented with $10 \%$ fetal calf serum, $1 \%$ non-essential aminoacids, $200 \mathrm{mM}$ L-glutamine and 1\% penicillin-streptomycin (all from Sigma Aldrich) to reach a final PVA concentration of $2.5 \%(\mathrm{w} / \mathrm{v})$. The PVA/G blend in CM was loaded inside 96-well culture plates, at $200 \mu \mathrm{L} / \mathrm{well}$, using sterile tips. After being filled with the solution, the well-plates were tightly closed to preserve the sterility and processed to obtain hydrogels via 
8 freeze-thawing cycles. The first cycle consisted of overnight freezing at $-20{ }^{\circ} \mathrm{C}$ followed by $30 \mathrm{~min}$ defrosting at room temperature. In the subsequent cycles $-20^{\circ} \mathrm{C}$ freezing was applied for $1 \mathrm{~h}$. At the end, the well-plate was opened under the cell culture flow cabinet and excess supernatant was removed. The gravimetric water content percent and the volume increase percent were calculated by weight and size measurements, with respect to wet and dry condition, respectively.

\subsection{Morphological Analysis of the PVA/G Hydrogels}

The specimens underwent preliminary dehydration through a graded ethanol/water solutions up to anhydrous ethanol that was removed using the Critical Point method (Balzers CPD030, Oerlikon Balzers, Balzers, Liechtenstein) to obtain dried hydrogels. The inner structure of the hydrogels was analyzed via SEM using a Jeol JSM-5600 LV (Jeol, Tokyo, Japan). The samples were mounted on aluminum stubs and coated with gold (Edwards Sputter Coater S150B, Edwards, NY, USA) prior to examination via SEM under an accelerating voltage of $10-15 \mathrm{kV}$.

Poral features of the hydrogels were investigated via mercury intrusion porosimetry $(n=3)$. Pore size ranging in $0.007-120 \mu \mathrm{m}$ diameters was evaluated by $\mathrm{Hg}$ intrusion using a porosimeter (Pascal 140, Carlo Erba, Pomezia, Italy) equipped with an automatic recording of intruded $\mathrm{Hg}$ volume. The pore volume distribution was obtained from the derivative curve of the cumulative intruded pore volume as function of pore diameter. This latter parameter is related to the measured pressure according to the Washburn's model equation developed for cylindrical shape pores [33]:

$$
d=10 \cdot 4 \gamma \cos \frac{\theta}{P}
$$

Stating that the cylindrical diameter $d(\mu \mathrm{m})$ of the $\mathrm{Hg}$-filled pores is inversely proportional to the intrusion pressure $P\left(\mathrm{~kg} / \mathrm{cm}^{2}\right)$, when the surface tension of $\mathrm{Hg} \gamma$ and the contact angle $\theta$ between $\mathrm{Hg}$ and the material are constant $(\gamma=0.48 \mathrm{~N} / \mathrm{m})$. Contact angles of $141.3^{\circ}$ were measured for these hydrogels.

\subsection{Cell Cultures}

The human hepatocarcinoma cell line HepG2 (ECACC, Sigma-Aldrich) was used to prepare cell/scaffold constructs. HepG2 cells were expanded using complete CM and passaged every 4-5 days using 1\% Trypsin/EDTA solution (Sigma-Aldrich) for detachment. For 2D studies, HepG2 were seeded on glass slides as multiple spots of $1 \times 10^{4}$ cells each, and let adhere for $2 \mathrm{~h}$, covered with complete CM and maintained in culture until the confluence was reached. Sterile PVA/G hydrogels were placed into 24-well plates and HepG2 cells were seeded into the scaffolds using a Hamilton syringe (Hamilton Bonaduz, Bonaduz, Switzerland) equipped with a thin needle (gauge 19).

Specifically, $1 \times 10^{6}$ cells were suspended in $20 \mu \mathrm{L}$ of complete $\mathrm{CM}$ and inoculated into the scaffold through multiple shots. Cells were let adhere for $2 \mathrm{~h}$ before adding $1 \mathrm{~mL}$ of complete $\mathrm{CM}$ inside the wells. All the cell cultures were conducted in a humidified incubator under standard conditions, namely, $37{ }^{\circ} \mathrm{C}, 95 \%$ humidity and $5 \% / 95 \% \mathrm{CO}_{2} /$ air mixture. 


\subsection{Viability of Cell/Scaffold Constructs}

Viability of HepG2 cells cultured inside PVA/gel hydrogels was evaluated using alamarBlue ${ }^{\circledR}$ assay (Life technologies, Carlsbad, CA, USA). Metabolically active cells are able to reduce the bioassay indicator resulting in color change of the CM. Moreover, samples can be assayed at different culture time-points thanks to the negligible toxicity of the dye. Data were acquired according to manufacturer instructions, and were expressed as percentage of reduced alamarBlue ${ }^{\circledR}(\% \mathrm{AB}$ red). Briefly, samples $(n=6)$ and controls, i.e., scaffolds with no cells $(n=6)$, were incubated for $3 \mathrm{~h}$ at $37{ }^{\circ} \mathrm{C}$ with the alamarBlue ${ }^{\circledR}$ dye diluted in CM according to the manufacturer's recommendations. Viability tests were performed every 7 days after seeding. At each time-point, $100 \mu \mathrm{L}$ of supernatant from sample or control was loaded in 96-well plates; then, excess supernatant was removed from the cultures and replaced with fresh CM. The absorbance $(\lambda)$ of supernatants was measured with a spectrophotometer (Microplate Reader Model 680, Biorad, Hercules, CA, USA) under a double wavelength reading (570 $\mathrm{nm}$ and $600 \mathrm{~nm})$.

Finally, \%ABred was calculated correlating the absorbance values and the molar extinction coefficients of the dye at the selected wavelengths, following the protocol provided by the manufacturer. The equation applied is shown below:

$$
\% \mathrm{AB}_{\text {red }}=100 \times \frac{\left(117,216 \cdot \lambda_{\text {sample@570nm }}-80,586 \cdot \lambda_{\text {sample@600nm }}\right)}{\left(155,677 \cdot \lambda_{\text {control@600nm }}-14,652 \cdot \lambda_{\text {control@570nm }}\right)}
$$

\subsection{Tumor Specimens}

Tumor specimens of three patients affected by HCC with no evidences of cirrhosis or fibrosis could be accessed in the framework of a retrospective study on hepatic tumors conducted by the Anatomic Pathology Unit at the Hospital of Cisanello, Pisa, Italy. Sections, 3- $\mu \mathrm{m}$ thick, were collected and treated for histochemistry and immunohistochemistry.

\subsection{Histologic Processing of Cell/Scaffold Constructs}

At the end of the culture, samples were treated for histologic analysis. Briefly, both cell/scaffolds constructs and plain scaffolds were fixed in $4 \%$ neutral buffered formalin $(0.1 \mathrm{M}, \mathrm{pH} 7.2)$ at $4{ }^{\circ} \mathrm{C}$ overnight, washed in $1 \times$ phosphate saline buffer (PBS; Sigma-Aldrich) four times for 15 min and finally rinsed in $70 \%$ ethanol until processing. Thereafter, the samples were dehydrated through a series of graded ethanol: $80 \%$ and $90 \%$ ethanol for $30 \mathrm{~min}$, 95\% ethanol for $60 \mathrm{~min}$ and absolute ethanol for $6 \mathrm{~h}$. Subsequently, the samples were clarified in xylene twice for $2 \mathrm{~h}$, rinsed in liquid paraffin at $60{ }^{\circ} \mathrm{C}$ for $4 \mathrm{~h}$ and wax embedded. Specimen sections $5-\mu \mathrm{m}$ thick were obtained, mounted onto slides and stored until histologic processing.

\subsection{H\&E Staining}

The sections were deparaffinized by immersion in xylene twice for $7 \mathrm{~min}$ and in absolute ethanol three times for $7 \mathrm{~min}$, stained with hematoxylin (Sigma-Aldrich) for $5 \mathrm{~min}$, washed in tap water for $5 \mathrm{~min}$, counterstained with eosin (Sigma-Aldrich) for $1 \mathrm{~min}$, then washed in tap water and dehydrated in 
95\% ethanol for $5 \mathrm{~min}$ and in absolute ethanol three times for $7 \mathrm{~min}$. After clarification in xylene three times for $7 \mathrm{~min}$, the specimens were mounted with DPX mountant medium (Fluka, Buchs, Switzerland).

\subsection{PAS Reaction}

After deparaffination and washing in dd- $\mathrm{H}_{2} \mathrm{O}$, the samples were incubated in $0.1 \%$ periodic acid in double-distilled $\mathrm{H}_{2} \mathrm{O}$ (Fluka) for $10 \mathrm{~min}$, air dried and treated with Schiff reagent (Sigma-Aldrich) for $15 \mathrm{~min}$. After washing in tap water for $10 \mathrm{~min}$, the sections were counterstained with hematoxylin for 5 min, washed in tap water for 5 min, dehydrated and mounted in DPX medium.

\subsection{Immunohistochemistry}

Specimen sections were deparaffinized and brought up to water; samples permeabilization was performed using $0.2 \%$ Triton X-100 (Sigma-Aldrich) in $1 \times$ PBS for $10 \mathrm{~min}$. Quenching of peroxidase was reached by incubation in $0.6 \% \mathrm{H}_{2} \mathrm{O}_{2}$ in methanol in the dark for $15 \mathrm{~min}$. In order to block the aspecific binding sites, the samples were incubated with goat serum (Vektor Lab, Burlingame, CA, USA) diluted 1:20 in $1 \times \mathrm{PBS}$ at $37^{\circ} \mathrm{C}$ for $20 \mathrm{~min}$. After washing, the samples were incubated with anti $\beta$-actin mouse monoclonal antibody (A-5316, Sigma-Aldrich) and anti-integrin $\alpha 5$ antibody (AB1928, Millipore, Merck KGaA, Darmstadt, Germany) diluted 1:2000 and 1:100, respectively, in $0.1 \% \mathrm{BSA} / 1 \times \mathrm{PBS}$ in moist chamber at $4{ }^{\circ} \mathrm{C}$ overnight. Negative controls were performed incubating some sections with $0.1 \%$ BSA/1 $\times$ PBS only. Next day, the samples were incubated with a goat anti-mouse biotinylated secondary antibody (Vektor Lab) diluted 1:200 in 1.5\% goat serum/1× PBS solution for 60 min and then with streptavidin (Vectastain Elite ABC Kit Standard, Vektor Lab) for $30 \mathrm{~min}$. In order to reveal the reaction, the sections were incubated in the chromogenic substrate solution at $0.5 \mathrm{mg} / \mathrm{mL} \mathrm{3,3'-diaminobenzidine}$ tetrahydrochloride containing $0.02 \% \mathrm{H}_{2} \mathrm{O}_{2}$ (Amresco, Solon, $\mathrm{OH}$, USA) for $5 \mathrm{~min}$ in the dark, counterstained with hematoxylin for $30 \mathrm{~s}$ and washed in tap water for $1 \mathrm{~min}$. Finally, the sections were dehydrated and mounted as previously described. In the second part of reaction, after each passage, washing in $0.01 \%$ Triton $/ 1 \times$ PBS was performed. The treated histologic sections were observed with a DMRB Leica microscope (Leica Microsystems, Wetzlar, Germany).

\subsection{Western Blotting}

At the endpoint of the culture, both cell/scaffolds constructs $(n=3)$ and controls (scaffolds without cells as a negative control, and cells grown in monolayer up to $80 \%$ confluence -4 days - as a positive control) were washed in D-PBS and resuspended in a cell lysis buffer (50 mM Tris-HCl, $\mathrm{pH} 7.4$, $150 \mathrm{mM} \mathrm{NaCl}, 2 \mathrm{mM}$ EDTA, 1\% NP-40, and antiproteases 1×, all from Sigma Aldrich). Samples were then disrupted using two $30 \mathrm{~s}$ pulses by Micro-Ultrasonic Cell Disrupter (Vineland, NJ, USA) set at 50\% output in an ice bath. Sample lysates were centrifuged at $15,000 \mathrm{rpm}$ for $20 \mathrm{~min}$ at $4{ }^{\circ} \mathrm{C}$ and protein concentration in the supernatants was determined by the bicinchoninic acid (BCA; Pierce, Rockford, IL, USA) microplate method. Proteins ( $30 \mu \mathrm{g} / \mathrm{lane}$ ) were separated on a $4 \%-15 \%$ polyacrylamide gel (BioRad, Hercules, CA, USA) under reducing conditions, transferred to a nitrocellulose membrane (Trans Turbo Blot system, BioRad). The membrane was blocked with $4 \%$ dry non-fat milk in $0.1 \%$ Tween/Tris Buffer Saline (T-TBS) and incubated overnight at $4{ }^{\circ} \mathrm{C}$ with anti $\beta$-actin mouse monoclonal antibody (A-5316, 
Sigma-Aldrich) or anti-HPRT rabbit monoclonal antibody (ab109021, Abcam, Cambridge, UK) diluted 1:2000 or 1:10,000 in T-TBS, respectively. Anti-mouse or anti-rabbit Horse Radish Peroxidase (HRP)-conjugated antibodies (KPL, Gaithersburg, MD, USA), both diluted 1:2000 in 4\% dry non-fat milk in T-TBS for $1 \mathrm{~h}$ at room temperature, were used as secondary antibodies and the immunocomplexes were detected by chemiluminescence (ECL clarity, BioRad) using Chemi-Doc XRS+ (BioRad). The data were analyzed using Image Lab software (BioRad).

\subsection{Statistical Analysis}

Quantitative data were presented as descriptive [mean \pm standard deviation (SD)] and inferential statistics ( $p$ values). Statistical significance was evaluated with two-tailed $t$-test for paired data, followed by Bonferroni correction. Significance was set at $p<0.05$.

\section{Conclusions}

This study was aimed at developing a new 3D in vitro model of $\mathrm{HCC}$ usable at late time-points to investigate tumor cell migration within a 3D environment. Specifically, a preliminary morphological investigation was performed to disclose morphological changes occurring in HepG2 cells grown for 24 days inside PVA/G hydrogels. From a morphological point of view, these bioartificial scaffolds showed anisotropic lamellar structures that reminded the trabecular structure of liver parenchyma. The cell/scaffold constructs were compared to HepG2 monolayers and tumor specimens via histology. In the 3D models, HepG2 cells were metabolically active, as demonstrated by glycoprotein synthesis, alamarBlue $^{\circledR}$ assay and HPRT expression. Large cellular aggregates were observed showing different morphotypes with zonal distribution associated to a peculiar $\beta$-actin and $\alpha 5 \beta 1$ immunolocalization. Specifically, the frontline cells were morphologically different from the inner cells and characterized by strong immunoreactions for both proteins, localized on a side border of their membrane, thus suggesting the formation of lamellipodia-like structures apt for migration. This study proposes PVA/G hydrogels as user-friendly and valuable substrates to develop long term 3D HCC models that can be useful to investigate important aspects of tumor biology related to cell migration and metastasis.

\section{Acknowledgments}

S.M. and S.D. wish to acknowledge Lorenzo P. Serino (University of Pisa, Italy) for his technical support in PVA/G hydrogel preparation and characterization, and Sabrina Danti (University of Pisa, Italy) for her contribute to statistical analysis.

\section{Author Contributions}

S.M. and S.D. conceived and designed the experiments; S.M. and F.R. performed the experiments; S.M., D.C. and S.D. analyzed the data; F.R. and D.C. contributed reagents/materials/analysis tools; S.M. and S.D. wrote the paper.

\section{Conflicts of Interest}

The authors declare no conflict of interest. 


\section{References}

1. Jemal, A.; Bray, F.; Center, M.M.; Ferlay, J.; Ward, E.; Forman, D. Global cancer statistics. CA Cancer J. Clin. 2011, 61, 69-90.

2. Bruix, J.; Sherman, M. Management of hepatocellular carcinoma: An update. Hepatology 2011, 53, 1020-1022.

3. Zhai, B.; Sun, X.Y. Mechanisms of resistance to sorafenib and the corresponding strategies in hepatocellular carcinoma. World J. Hepatol. 2013, 5, 345-352.

4. Berasain, C. Hepatocellular carcinoma and sorafenib: Too many resistance mechanisms? Gut 2013, $62,1674-1675$.

5. Holbeck, S.L. Update on NCI in vitro drug screen utilities. Eur. J. Cancer 2004, 40, 785-793.

6. Voskoglou-Nomikos, T.; Pater, J.L.; Seymour, L. Clinical predictive value of the in vitro cell line, human xenograft, and mouse allograft preclinical cancer models. Clin. Cancer Res. 2003, 9, 4227-4239.

7. Ricci, C.; Moroni, L.; Danti, S. Cancer tissue engineering-New perspectives in understanding the biology of solid tumors-A critical review. OA Tissue Eng. 2013, 1, 4:1-4:7.

8. Fischbach, C.; Chen, R.; Matsumoto, T.; Schmelzle, T.; Brugge, J.S.; Polverini, P.J.; Mooney, D.J. Engineering tumors with 3D scaffolds. Nat. Methods 2007, 4, 855-860.

9. Yamada, K.M.; Cukierman, E. Modeling tissue morphogenesis and cancer in 3D. Cell 2007, 130, 601-610.

10. Xu, X.; Farach-Carson, M.C.; Jia, X. Three-dimensional in vitro tumor models for cancer research and drug evaluation. Biotechnol. Adv. 2014, 32, 1256-1268.

11. Schmeichel, K.L.; Bissell, M.J. Modeling tissue-specific signaling and organ function in three dimensions. J. Cell Sci. 2003, 116, 2377-2388.

12. Horiuchi, S.; Ishida, S.; Hongo, T.; Ishikawa, Y.; Miyajima, A.; Sawada, J.; Ohno, Y.; Nakazawa, K.; Ozawa, S. Global gene expression changes including drug metabolism and disposition induced by three-dimensional culture of HepG2 cells-Involvement of microtubules. Biochem. Biophys. Res. Commun. 2009, 378, 558-562.

13. Bokhari, M.; Carnachan, R.J.; Cameron, N.R.; Przyborski, S.A. Culture of HepG2 liver cells on three dimensional polystyrene scaffolds enhances cell structure and function during toxicological challenge. J. Anat. 2007, 211, 567-576.

14. Chang, T.T.; Hughes-Fulford, M. Monolayer and spheroid culture of human liver hepatocellular carcinoma cell line cells demonstrate distinct global gene expression patterns and functional phenotypes. Tissue Eng. Part A 2009, 559-567.

15. Kulig, K.M.; Vacanti, J.P. Hepatic tissue engineering. Transpl. Immunol. 2004, 12, 303-310.

16. Hutmacher, D.W.; Loessner, D.; Rizzi, S.; Kaplan, D.L.; Mooney, D.J.; Clements, J.A. Can tissue engineering concepts advance tumor biology research? Trends Biotechonol. Rev. 2010, 28, 125-133.

17. Kataoka, K.; Nagao, Y.; Nukui, T.; Akiyama, I.; Tsuru, K.; Hayakawa, S.; Osaka, A.; Huh, N. An organic-inorganic hybrid scaffold for the culture of HepG2 cells in a bioreactor. Biomaterials 2005, 26, 2509-2516.

18. Kinasiewicz, A.; Kawiak, J.; Weryńskia, A. 3D matrigel culture improves differentiated functions of HepG2 cells in vitro. Biocybern. Biomed. Eng. 2006, 26, 47-55. 
19. Wu, M.; Yang, Z.; Liu, Y.; Liu, B.; Zhao, X. The 3-D culture and in vivo growth of the human hepatocellular carcinoma cell line HepG2 in a self-assembling peptide nanofiber scaffold. J. Nanomater. 2010, 2010, 1-7.

20. Lan, S.-F.; Safiejko-Mroczka, B.; Starly, B. Long-term cultivation of HepG2 liver cells encapsulated in alginate hydrogels: A study of cell viability, morphology and drug metabolism. Toxicol. In Vitro 2010, 24, 1314-1323.

21. Lee, K.Y.; Mooney, D.J. Hydrogels for tissue engineering. Chem. Rev. 2001, 101, 1869-1879.

22. Drury, J.; Mooney, D. Hydrogels for tissue engineering: Scaffold design variables and applications. Biomaterials 2003, 24, 4337-4351.

23. Thankam, F.G.; Muthu, J. Biosynthetic hydrogels - Studies on chemical and physical characteristics on long-term cellular response for tissue engineering. J. Biomed. Mater. Res. Part A 2014, 102, 2238-2247.

24. Hassan, C.; Peppas, N.A. Structure and morphology of freeze/thawed PVA hydrogels. Macromolecules 2000, 33, 2472-2479.

25. Cascone, M.G.; Lazzeri, L.; Sparvoli, E.; Scatena, M.; Serino, L.P.; Danti, S. Morphological evaluation of bioartificial hydrogels as potential tissue engineering scaffolds. J. Mater. Sci. Mater. Med. 2004, 15, 1309-1313.

26. Loh, Q.L.; Choong, C. Three-dimensional scaffolds for tissue engineering applications: Role of porosity and pore size. Tissue Eng. Part B Rev. 2013, 19, 485-502.

27. Ricci, C.; Mota, C.; Moscato, S.; D’Alessandro, D.; Ugel, S.; Sartoris, S.; Bronte, V.; Boggi, U.; Campani, D.; Funel, N.; et al. Interfacing polymeric scaffolds with primary pancreatic ductal adenocarcinoma cells to develop 3D cancer models. Biomatter 2014, 4, e955386:1-e955386:11.

28. Moscato, S.; Mattii, L.; D’Alessandro, D.; Cascone, M.G.; Lazzeri, L.; Serino, L.P.; Dolfi, A.; Bernardini, N. Interaction of human gingival fibroblasts with PVA/gelatine sponges. Micron 2008, 39, 569-579.

29. Mueller-Klieser, W. Multicellular spheroids: A review on cellular aggregates in cancer research. J. Cancer Res. Clin. Oncol. 1987, 113, 101-122.

30. Burdett, E.; Kasper, F.K.; Mikos, A.J.; Ludwig, J.A. Engineering tumors: A tissue engineering perspective in cancer biology. Tissue Eng. Part B Rev. 2006, 12, 3265-3283.

31. Grimes, D.R.; Kelly, C.; Bloch, K.; Partridge, M. A method for estimating the oxygen consumption rate in multicellular tumour spheroids. J. R. Soc. Interface 2013, doi:10.1098/rsif.2013.1124.

32. Yamaguchi, H.; Condeelis, J. Regulation of the actin cytoskeleton in cancer cell migration and invasion. Biochim. Biophys. Acta 2007, 1773, 642-652.

33. Washburn, E.W. Note on a method of determining the distribution of pore sizes in a porous material. Proc. Natl. Acad. Sci. USA 1921, 7, 115-116.

(C) 2015 by the authors; licensee MDPI, Basel, Switzerland. This article is an open access article distributed under the terms and conditions of the Creative Commons Attribution license (http://creativecommons.org/licenses/by/4.0/). 